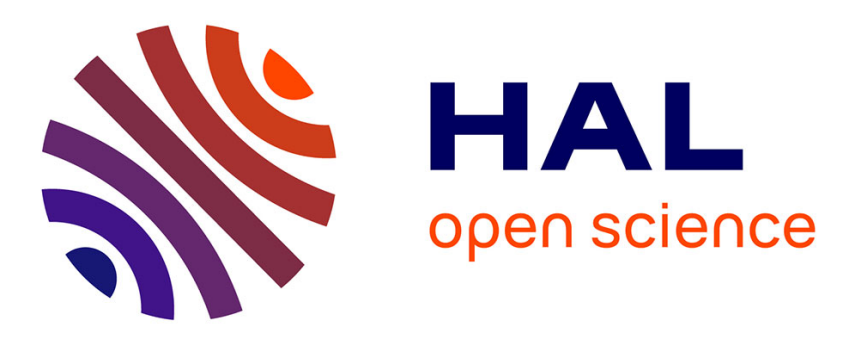

\title{
Thermal activation effects on the exchange bias in ferromagnetic-antiferromagnetic nanostructures
}

\author{
Vincent Baltz, J. Sort, B. Rodmacq, B. Dieny, S. Landis
}

\section{To cite this version:}

Vincent Baltz, J. Sort, B. Rodmacq, B. Dieny, S. Landis. Thermal activation effects on the exchange bias in ferromagnetic-antiferromagnetic nanostructures. Physical Review B: Condensed Matter and Materials Physics (1998-2015), 2005, 72, pp.104419. 10.1103/PhysRevB.72.104419 . hal-01683704

\section{HAL Id: hal-01683704 https://hal.science/hal-01683704}

Submitted on 25 May 2019

HAL is a multi-disciplinary open access archive for the deposit and dissemination of scientific research documents, whether they are published or not. The documents may come from teaching and research institutions in France or abroad, or from public or private research centers.
L'archive ouverte pluridisciplinaire HAL, est destinée au dépôt et à la diffusion de documents scientifiques de niveau recherche, publiés ou non, émanant des établissements d'enseignement et de recherche français ou étrangers, des laboratoires publics ou privés. 


\title{
Thermal activation effects on the exchange bias in ferromagnetic-antiferromagnetic nanostructures
}

\author{
V. Baltz,* J. Sort, B. Rodmacq, and B. Dieny \\ SPINTEC (URA 2512), CEA/CNRS, 17 Avenue des Martyrs, 38054 Grenoble Cedex 9, France \\ S. Landis \\ LETI/D2NT, CEA, 17 Avenue des Martyrs, 38054 Grenoble Cedex 9, France \\ (Received 20 April 2005; revised manuscript received 8 July 2005; published 9 September 2005)
}

\begin{abstract}
The hysteresis loop shift $H_{E}$ of sub-100-nm ferromagnetic- (FM-) antiferromagnetic (AFM) nanostructures is found to be strongly influenced by thermal activation effects. These effects, which tend to reduce $H_{E}$, are more pronounced in the nanostructures than in continuous films with the same composition, particularly for thin AFM layers. In addition, the reduced dimensions of the nanostructures also impose spatial constraints to the AFM domain size, particularly for thick AFM layers. This favors an enhancement of $H_{E}$. Due to the interplay between these two competing effects, the loop shift in the dots can be either larger or smaller than in the continuous films with the same composition, depending on both the AFM thickness and temperature. A temperature-AFM thickness phase diagram, separating the conditions resulting in larger or smaller $H_{E}$ in the nanostructures with respect to continuous film is derived.
\end{abstract}

DOI: 10.1103/PhysRevB.72.104419

PACS number(s): 75.75.+a, 75.60.-d

\section{INTRODUCTION}

The exchange interactions between ferromagnetic (FM) and antiferromagnetic (AFM) materials typically result in a shift of the hysteresis loop, along the magnetic field axis $\left(H_{E}\right)$, referred to as exchange bias (EB), which is usually accompanied with an enhancement of the coercivity $\left(H_{C}\right){ }^{1}$ These effects are often ascribed to the formation of AFM domains which result in AFM uncompensated interfacial spins. During magnetization reversal, strongly pinned AFM uncompensated spins give rise to the loop shift, whereas unpinned spins are mainly responsible for the coercivity enhancement. $^{2-5}$ Upon heating, a decrease of $H_{E}$ is observed since the anisotropy and exchange energies in the AFM have to compete with thermal energy, which tends to reduce the stability of the AFM spin lattice and, consequently, the pinning strength that the AFM exerts on the FM. The temperature at which the exchange bias completely disappears is called the blocking temperature $\left(T_{B}\right)$ and is below the Néel temperature of the AFM (particularly for thin AFM).

During the last decades, a large variety of applications such as magnetoresistive read heads or magnetic random access memories (MRAMs) have triggered the study of EB properties. ${ }^{6}$ The constant miniaturization of devices ${ }^{7}$ has recently motivated studies of EB properties in systems with reduced lateral dimensions. ${ }^{8-24}$ Such investigation of confined EB systems down to length scales comparable to the AFM domains sizes is crucial for a better understanding of the EB phenomenon, since it allows probing the role of AFM domains on EB. However, the effects of confinement, such as the alteration of the AFM domain structure in nanostructures, have been far less explored. In particular, EB was investigated in nanostructures whose lateral sizes were varied, while keeping the AFM thickness constant (i.e., twodimensional confinement of the AFM) ${ }^{8,10-12,15,16,18}$ Occasionally, the effects of the temperature on the EB properties of nanostructures have also been reported. ${ }^{9,11,12}$ It is notewor- thy that some of the results from different groups seem contradictory. Namely, although in some systems $H_{E}$ is found to increase in the nanostructures with respect to the continuous film, ${ }^{8-11}$ the opposite behavior is encountered in other systems. ${ }^{12-22}$ We have recently shown that, at room temperature, for a given dot size, either an enhancement or a reduction of $H_{E}$ can be obtained in the FM-AFM dots, depending on the AFM thickness. ${ }^{23}$ Thermal activation is found to play a crucial role on this effect. Many experimental ${ }^{25-32}$ and theoretical $^{30-35}$ studies have been performed on continuous films to better understand thermal activation effects (e.g., after effects, blocking temperature distributions, ...) on the EB properties. Surprisingly, although thermal activation plays a role in EB properties, few systematic studies on these effects have been carried out in nanostructures..$^{11,12}$

In this article, we report on thermal activation effects on the EB of sub-100-nm FM-AFM bilayers sputtered on prepatterned $\mathrm{Si} / \mathrm{SiO}_{2}$ square dots. We demonstrate that systems with nanostructured and/or thinner AFM layers are more prone to thermal activation effects than continuous and/or thicker AFM layers. From the interplay between thermal activation effects (which reduce $H_{E}$ ) and the physically imposed spatial constraints on the AFM domain size (which can induce an enhancement of $H_{E}$ ), we show that the magnitude of the hysteresis loop shift $H_{E}$ in the nanostructures can be either larger or smaller than in continuous films with the same composition, depending on both the AFM thickness and temperature conditions.

\section{EXPERIMENT}

The nanostructures were fabricated by first patterning thermally oxidized $\mathrm{Si}$ wafers by electron beam lithography and reactive ion etching to form arrays of $\mathrm{Si} / \mathrm{SiO}_{2}$ square dots, with lateral sizes of $90 \mathrm{~nm}$, height of $300 \mathrm{~nm}$, and periodicity of $200 \mathrm{~nm}$, as shown in Fig. 1. A series of samples consisting of stacks of 


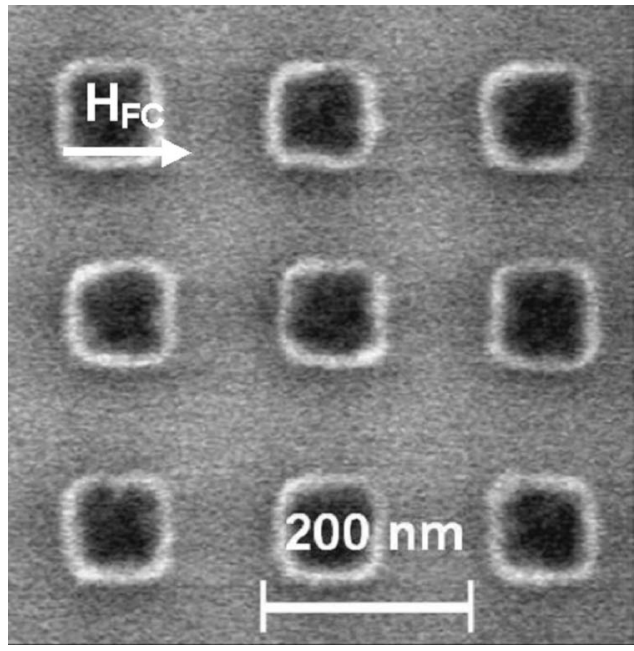

FIG. 1. Scanning electron microscopy (SEM) image of the array of $90 \times 90 \mathrm{~nm}^{2}$ square dots fabricated by first patterning a $\mathrm{Si} / \mathrm{SiO}_{2}$ wafer and subsequently depositing the FM-AFM bilayer structure.

$\operatorname{Ta}(5 \mathrm{~nm}) / \operatorname{Py}(12 \mathrm{~nm}) / \operatorname{IrMn}\left(t_{\mathrm{IrMn}}\right) / \operatorname{Pt}(2 \mathrm{~nm})$ (where Py denotes permalloy, i.e., $\mathrm{Ni}_{81} \mathrm{Fe}_{19}$, which is $\mathrm{FM}$ and $\mathrm{IrMn}$ stands for $\operatorname{Ir}_{20} \mathrm{Mn}_{80}$, which is AFM) were simultaneously dc sputtered, on continuous and patterned $\mathrm{Si} / \mathrm{SiO}_{2}$ wafers. The Ta and $\mathrm{Pt}$ are, respectively, buffer and capping layers. All depositions were performed at room temperature under a $2.5 \mathrm{~Pa}$ Ar pressure with deposition rates around $0.08 \mathrm{~nm} \mathrm{~s}^{-1} .^{36}$ The IrMn thickness $t_{\mathrm{IrMn}}$ was varied from 5 to $19 \mathrm{~nm}$. It should be noted that the direct deposition of the magnetic material on the already prepatterned substrates allows avoiding possible degradation effects due to post-deposition etching, such as partial structural deterioration of the layers. ${ }^{36,37}$

The EB was set by post annealing and field cooling the samples in a $5 \times 10^{-3} \mathrm{~Pa}$ vacuum, from $T=550 \mathrm{~K}$ (i.e., from above the blocking temperature $T_{B}$ of all systems ${ }^{23}$ ) in a $2.4 \mathrm{kOe}$ in-plane magnetic field, applied parallel to one of the sides of the square dots, as indicated in Fig. 1. Hysteresis loops were recorded at room temperature by longitudinal magneto-optical Kerr effect (MOKE) with a field sweep rate of about $1 \mathrm{Oe} \mathrm{s}^{-1}$ and at low temperatures by SQUID magnetometry with a lower field sweep rate around $0.03 \mathrm{Oe} \mathrm{s}^{-1}$. The MOKE and SQUID data are both obtained by a single hysteresis loop (i.e., without averaging loops). Virtually no training effect was observed except a slight $15 \%$ loss in the magnitude of $H_{E}$ for the nanostructures with $t_{\mathrm{IrMn}}=5 \mathrm{~nm}$ between the first and second loops. Care was taken for the 5 -nm IrMn thick nanostructures, where the second loops were plotted and used. It should be noted that the pronounced shadowing effects resulting from the combination of the pillars height to lateral spacing ratio $(300 / 110)$, together with the low incidence angle $\left(30^{\circ}\right)$ used for the Kerr measurements, allow masking the signal from the trenches when performing hysteresis loops using MOKE. However, both the signal from the dots and from the trenches are simultaneously detected when using SQUID.

Thermal activation at high temperatures was studied by applying a commonly used procedure, ${ }^{30}$ which consists in (i) field cooling the samples under a positive field, $H_{\mathrm{FC}}$ $=2.4 \mathrm{kOe}$ from above $T_{B}\left(T_{\text {heat }}=550 \mathrm{~K}\right)$, (ii) heating the samples again but now to a lower temperature $\left(T_{\text {heat } 2}<T_{B}\right)$ and field cooling them to room temperature, using a negative field, $H_{\mathrm{FC}}=-2.4 \mathrm{kOe}$, (iii) measuring the hysteresis loop after the two consecutive field cooling procedures at room temperature. It is noteworthy that all the heating and cooling processes were performed under a $5 \times 10^{-3} \mathrm{~Pa}$ vacuum. This procedure allows probing high temperature thermal activation effects. ${ }^{30}$ Namely, at $T=T_{\text {heat } 2}$, if thermal activation effects are present, the initially induced exchange bias (i.e., with the first cooling in positive field) will be reduced due to partial loss of the AFM pinning strength. Indeed, due to local inhomogeneities in the FM-AFM interface (roughness, defects, ...) or variations in the AFM crystallite sizes, a distribution of local blocking temperatures in FM-AFM systems is typically encountered. ${ }^{1}$ Since the samples are cooled again using a negative field, the AFM spins affected by the thermal activation at $T_{\text {heat2 }}$ will then be realigned but in the opposite direction, leading to an overall reduction of $H_{E}$ when measured at room temperature after the two consecutive cooling procedures. Hence, it is possible to extract information about the thermal activation effects occurring at $T=T_{\text {heat2 }}$ from the measurement at room temperature of the hysteresis loop after the two consecutive cooling procedures.

\section{RESULTS AND DISCUSSION}

Figure 2 shows hysteresis loops performed by MOKE, at room temperature, along the field cooling direction of (a) the continuous films and (b) the nanostructures, with compositions $\mathrm{Ta}(5 \mathrm{~nm}) / \mathrm{Py}(12 \mathrm{~nm}) / \operatorname{IrMn}(5 \mathrm{~nm}) / \operatorname{Pt}(2 \mathrm{~nm})$, after field cooling from different temperatures $\left(T_{\text {heat } 2}=298,328\right.$, and $343 \mathrm{~K}$ ). It can be observed that the value of $H_{E}$ progressively decreases with $T_{\text {heat } 2}$ both in the continuous film and in the nanostructures. This is due to the enhanced thermal acti- (a)continuous film

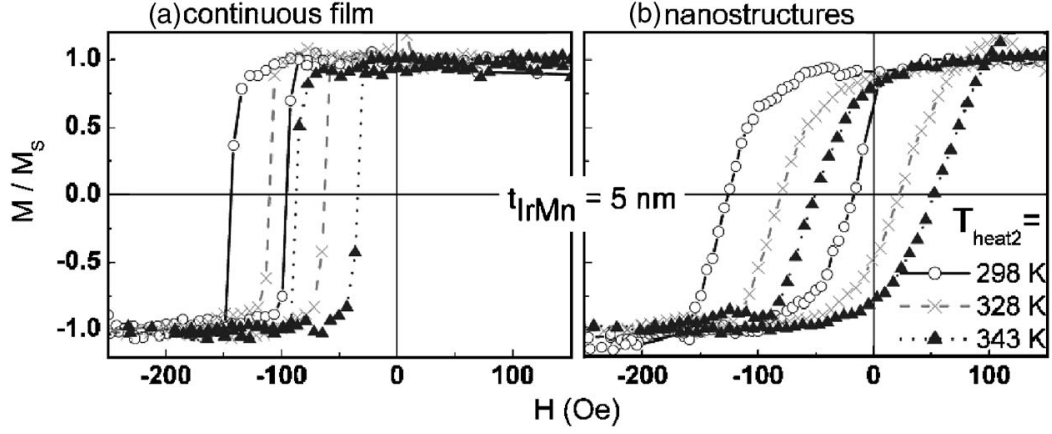

FIG. 2. Hysteresis loops of (a) the continuous films and (b) the nanostructures, with compositions $\quad \mathrm{Ta}(5 \mathrm{~nm}) / \mathrm{Py}(12 \mathrm{~nm}) / \operatorname{IrMn}(5 \mathrm{~nm}) /$ $\operatorname{Pt}(2 \mathrm{~nm})$, for different temperatures $\left[T_{\text {heat } 2}\right.$ $=298 \mathrm{~K}\left(-\mathrm{O}_{-}\right), 328 \mathrm{~K}(-\mathrm{x}-)$, and $343 \mathrm{~K}\left(-\boldsymbol{\Delta}_{-}\right)$] from which the continuous film and the nanostructures, previously field cooled in $H_{\mathrm{FC}}$ $=2.4 \mathrm{kOe}$ from above $T_{B}$, have been field cooled again in $H_{\mathrm{FC}}=-2.4 \mathrm{kOe}$. All measurements have been performed at room temperature by longitudinal Kerr effect along the field cooling direction. 
vation at higher temperatures. Namely, if the AFM layer is sufficiently thin (e.g., $5 \mathrm{~nm}$ ) an increasing proportion of the AFM spin lattice becomes unpinned when the temperature is raised to $T_{\text {heat } 2}$ (AFM spins which become pinned again during the second field cooling in the direction opposite to that initially set during the first field cooling).$^{30}$ Contrary to $H_{E}$, the coercivity seems to be independent on $T_{\text {heat } 2}$ for both the continuous films and the nanostructures. It is noteworthy that $H_{C}$ of the dots is larger than in the continuous films $\left(H_{C \text {,dots }} \sim 55 \mathrm{Oe}\right.$ and $\left.H_{C \text {,cont }} \sim 30 \mathrm{Oe}\right)$. This trend is often encountered when comparing continuous films and nanostructures with the same composition (even without the presence of the AFM) and is mainly ascribed to the reduced number of nucleation centers and to enhanced pinning of domain walls at the edges of the nanostructures. ${ }^{37,38}$ Additional loss in the domain wall motion is expected in FM-AFM EB unpatterned and patterned bilayers. ${ }^{1,39}$ In unpatterned EB systems losses due to FM domain wall motion can significantly contribute to the coercivity, particularly at temperatures well below the blocking temperature. As will be further discussed, possible changes in the magnetostatic energy of the FM in EB nanostructures might account for enhanced coercivity with respect to the continuous film. ${ }^{40}$ Moreover, in EB bilayers more pronounced dragging of the AFM spins in the nanostructures can account for an additional coercivity enhancement. ${ }^{1,12,14}$ It is noteworthy that although the cooling procedure allows probing high temperature activation effects on $H_{E}$, thermal effects on $H_{C}$ cannot be probed by using this particular heating procedure, i.e., after field cooling the system twice, using fields of opposite sign. ${ }^{30}$ Indeed, $H_{C}$ is related to AFM spins which are dragged during the magnetization reversal of the FM layer. ${ }^{5}$ During the heating to $T_{\text {heat }}$, the pinning strength exerted by the AFM spins is altered. However, once back to room temperature, this pinning recovers its initial strength. As a result, $H_{C}$ remains independent of the heating temperature $T_{\text {heat } 2}$. Furthermore, Fig. 2 reveals that for $T_{\text {heat } 2}=343 \mathrm{~K}, H_{E}$ in the nanostructures has almost vanished although $H_{E}$ in the continuous film is still rather large $\left(H_{E, \text { cont }} \sim 60 \mathrm{Oe}\right)$. This indicates that thermal activation effects at this temperature are much larger in the nanostructures than in the continuous films. It is also noteworthy that the loops corresponding to the nanostructures are somewhat more slanted than those corresponding to the continuous films. This loop shearing is ascribed to shape inhomogeneities among the dots, which typically result in a broadening of the switching field distribution. ${ }^{36}$ Compared to other studies of EB in patterned elements, ${ }^{9,19,20}$ no pronounced asymmetries are observed in our case.

Figure 3(a) shows the evolution of $H_{E}$, estimated from the hysteresis loops measured at room temperature, on $T_{\text {heat } 2}$, for both the continuous films and the nanostructures with composition $\mathrm{Ta}(5 \mathrm{~nm}) / \operatorname{Py}(12 \mathrm{~nm}) / \operatorname{IrMn}\left(t_{\mathrm{IrMn}}\right) / \operatorname{Pt}(2 \mathrm{~nm})$, with $t_{\mathrm{IrMn}}=5,9$, and $16 \mathrm{~nm}$. As already discussed, for a given composition the magnitude of $H_{E}$, measured at room temperature, progressively decreases with $T_{\text {heat2 }}$ and then changes sign since locally, regions of AFM spins with $T_{B}$ smaller than $T_{\text {heat } 2}$ progressively reverse direction during the second field cooling procedure. It is noteworthy that, for any given $t_{\mathrm{IrMn}}, H_{E}$ in the nanostructures tends to decrease faster with $T_{\text {heat } 2}$ than $H_{E}$ in the continuous films, thus evidencing
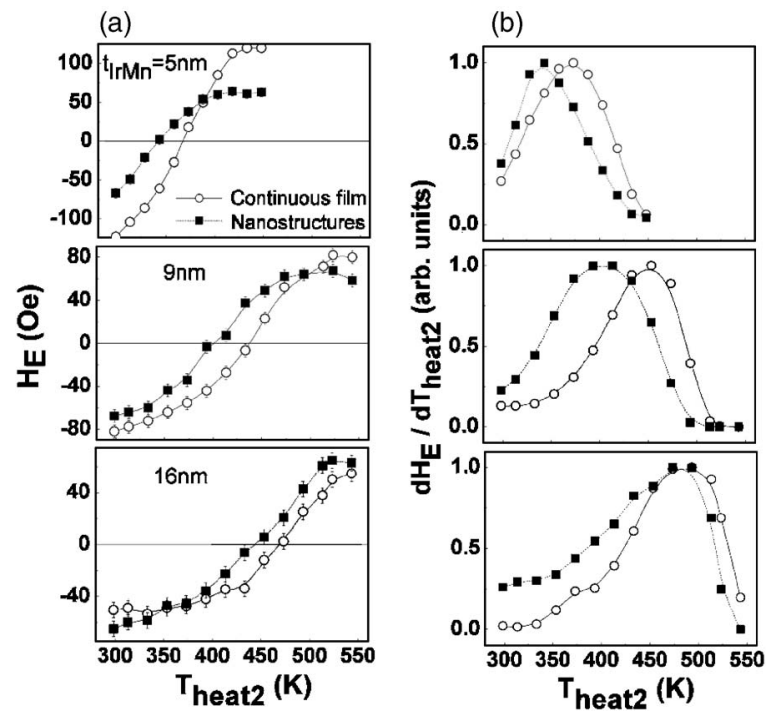

FIG. 3. (a) Dependence of the exchange bias field $H_{E}$ on the temperature $T_{\text {heat2 }}$ from which the continuous film (-O-) and the nanostructures (-口-), with compositions $\mathrm{Ta}(5 \mathrm{~nm}) / \mathrm{Py}(12 \mathrm{~nm}) /$ $\operatorname{IrMn}\left(t_{\mathrm{IrMn}}\right) / \operatorname{Pt}(2 \mathrm{~nm})$, with $t_{\mathrm{IrMn}}=5,9$, and $16 \mathrm{~nm}$, initially field cooled in $H_{\mathrm{FC}}=2.4 \mathrm{kOe}$ from above $T_{B}$, have been field cooled in

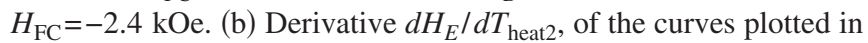
(a), which are typically representative of the blocking temperature distributions. The lines are guides to the eye.

that $H_{E}$ in the nanostructures is more subject to thermal activation. Moreover, $H_{E}$ in the nanostructures is found to vanish at lower temperatures $\left(T_{H E=0}\right)$ than in the continuous film. This means that the configuration where half of the AFM pinned uncompensated spins are oriented in one set direction and half in the opposite (i.e., where half of the regions have local blocking temperatures above $T_{H E=0}$ and half below) is observed at lower temperatures for the nanostructures. The heating temperature at which $H_{E}$ levels off after the second field cooling in a negative field [see Fig. 3(a)], represents the maximum local blocking temperature and is also found to be lower in the nanostructures. Moreover, the already reported $T_{B}$ reduction and/or $H_{C}$ enhancement in FM-AFM nanostructures ${ }^{8,9,12-15,23}$ corroborates the idea that thermal activation is indeed more pronounced in nanostructures than in continuous FM-AFM bilayers. The derivatives $d H_{E} / d T_{\text {heat2 }}$, which are plotted in Fig. 3(b) are typically used to represent the blocking temperature distribution of FMAFM bilayers. ${ }^{1}$ As can be seen in the figure, these $T_{B}$ distributions are overall shifted towards lower temperature values for the nanostructures. It can be argued that, due to their reduced coordination numbers, AFM spins located at the edges of the dots can be more easily dragged during the magnetization reversal of the FM, which might account for the weakening of the pinning strength and the enhanced thermal activation in the nanostructures. This can bring about an enhancement of $H_{C}$ in the nanostructures, which is also observed in our case (see Fig. 2).

Figure 3(a) shows as well that, for both continuous films and nanostructures, thin AFM layers are more clearly affected by thermal activation effects than thicker ones, since $H_{E}$ decreases faster with $T_{\text {heat2 }}$ for thinner AFM layers. This 
is in agreement with the often reported reduction of $T_{B}$ for low values of $t_{\mathrm{AFM}} \cdot 1,14,23,27,33$ This also manifests in a shift towards higher values of the blocking temperature distribution for thicker AFM layers, as can be seen in Fig. 3(b). It should be noted that a coherent columnar growth of the layers could be assessed from x-ray diffraction measurements. Namely, the vertical coherence lengths extracted from the widths of $\theta-2 \theta$ scan peaks showed that a good structural coherence exists within each grain throughout the AFM. As a result, for thicker AFM, the grains are expected to maintain the AFM order up to larger values of temperature. ${ }^{1}$ Note that the $T_{B}$ distributions also seem to slightly broaden for thicker AFM. The fact that for thicker IrMn layers thermal activation effects may be important over a slightly broader range of temperatures could be due to an increasing number of defects in the AFM layer, or to a broadening in the grain size distribution.

Figure 4 shows the evolution of $H_{E}$, estimated from the hysteresis loops measured at room temperature, on $t_{\mathrm{IrMn}}$ for both the continuous films and the nanostructures, after the first field cooling process using a positive field from $T_{\text {heat } 1}$ $=550 \mathrm{~K}$ [shown in Fig. 4(a), taken from Fig. 2 of Ref. 23] and after the subsequent field cooling under a negative field, from $T_{\text {heat } 2}=313,333$, and $353 \mathrm{~K}$ [shown in Figs. 4(b) $-4(\mathrm{~d})$, respectively]. After the first field cooling procedure, $H_{E}$ in the continuous films decreases as $t_{\mathrm{IrMn}}$ increases whereas $H_{E}$ in the nanostructures seems to be rather insensitive to $t_{\mathrm{IrMn}}$. As a result, although $H_{E}$ in the nanostructures is smaller than for the continuous films for thin AFM layers, larger $H_{E}$ in the dots is observed for $t_{\mathrm{IrMn}}$ larger than $12 \mathrm{~nm}^{23}$ These behaviors can be ascribed to the three-dimensional confinement of AFM domains in the nanostructures. Namely, the decrease of $H_{E}$ with the AFM thickness is often observed in continuous FM-AFM systems ${ }^{1,23,25,28,31}$ and can be attributed to the increase of the AFM domain size with the AFM thickness. Such inverse proportionality relationship between $H_{E}$ and the AFM thickness was already predicted by Malozemoff in his random field model for exchange bias, in the so-called Heisenberg regime ${ }^{3}$ and has been proved to be valid also in the framework of the so-called domain state model. ${ }^{31}$ According to Malozemoff's model, in a given interface area $L^{2}$ (which would correspond to the characteristic AFM domain size), containing $N$ atoms (so that $N=L^{2} / a^{2}$, where $a$ is the lattice constant), the local FM-AFM interface energy $\sigma_{l}$ $= \pm J / a^{2}$ (whose sign depends on the local orientation between the FM and AFM spins) averages statistically as $\sigma$ $=\sigma_{l} / N^{1 / 2}$ (thus being inversely proportional to $L$, the AFM domain size). For IrMn, using appropriate values of anisotropy and exchange constants, the domain size can be estimated to range between $160 \mathrm{~nm}$ for $t_{\mathrm{IrMn}}=5 \mathrm{~nm}$ and $620 \mathrm{~nm}$ for $t_{\mathrm{IrMn}}=19 \mathrm{~nm} \cdot{ }^{23}$ Hence, although the AFM domain size increases with $t_{\mathrm{IrMn}}$ for the continuous films, resulting in a reduction of $H_{E}$, its evolution is physically limited by the dots sizes (i.e., $90 \mathrm{~nm}$ ) in the nanostructures, thus maintaining a constant value of $H_{E}$ in the overall $t_{\text {IrMn }}$ range. As a result of the enhanced thermal activation in thin and/or nanostructured AFM, the reduction of $H_{E}$ with $t_{\mathrm{IrMn}}$ is smoothed for the continuous films as $T_{\text {heat } 2}$ increases, ${ }^{11}$ and, in contrast, $H_{E}$ starts to increase with $t_{\mathrm{IrMn}}$ for the nanostructures. It is noteworthy that assuming that AFM spins are more easily

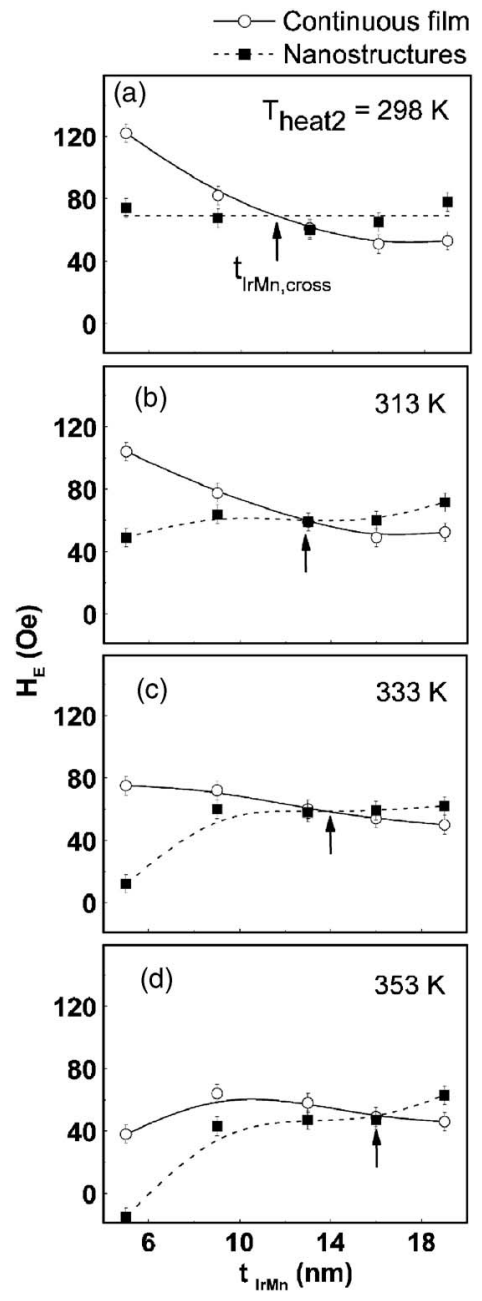

FIG. 4. Dependence of the exchange bias field $H_{E}$ on the IrMn thickness $t_{\mathrm{IrMn}}$ for both continuous films (- $\left.\bigcirc_{-}\right)$and nanostructures (-口-) with compositions $\mathrm{Ta}(5 \mathrm{~nm}) / \mathrm{Py}(12 \mathrm{~nm}) / \operatorname{IrMn}\left(t_{\mathrm{IrMn}}\right) /$ $\mathrm{Pt}(2 \mathrm{~nm}),(\mathrm{a})$ after a first field cooling $\left(H_{\mathrm{FC}}=2.4 \mathrm{kOe}\right)$ from $550 \mathrm{~K}$ and (b) $-(\mathrm{d})$ after a second field cooling $\left(H_{\mathrm{FC}}=-2.4 \mathrm{kOe}\right)$ from different heating temperatures $\left(T_{\text {heat } 2}=313,333\right.$, and $\left.353 \mathrm{~K}\right)$. The lines are guides to the eye. The arrows show the intersection of the evolutions in the nanostructures and in the continuous film. Note that Fig. 4(a) has been taken from Fig. 2 of Ref. 23.

unpinned due to thermal activation for thinner AFM layers, one might expect $H_{E}$ to increase with $t_{\mathrm{IrMn}}$ in the nanostructures, even at room temperature. This is not observed probably because room temperature is still rather low compared with the maximum blocking temperature for these compositions (e.g., $T_{B} \sim 390 \mathrm{~K}$ for $t_{\mathrm{IrMn}}=5 \mathrm{~nm}$ in the nanostructures $\left.{ }^{23}\right)$.

As can be seen in Fig. 4 the value of $t_{\mathrm{IrMn}}$ at which, for a given $T_{\text {heat } 2}$, the evolutions of $H_{E}$ on $t_{\mathrm{IrMn}}$ for the nanostructures and the continuous film intersect, $t_{\mathrm{IrMn} \text {,cross }}$ increases with $T_{\text {heat } 2 \text {. This means that for lower heating temperatures }}$ the exchange bias in the nanostructures is found to be larger than for the continuous films in a broader AFM thickness range. It should be noted that, due to thermal activation, even at heating temperatures larger than room temperature, thicker layers are required to obtain larger $H_{E}$ in the nanostructures than in continuous films. 


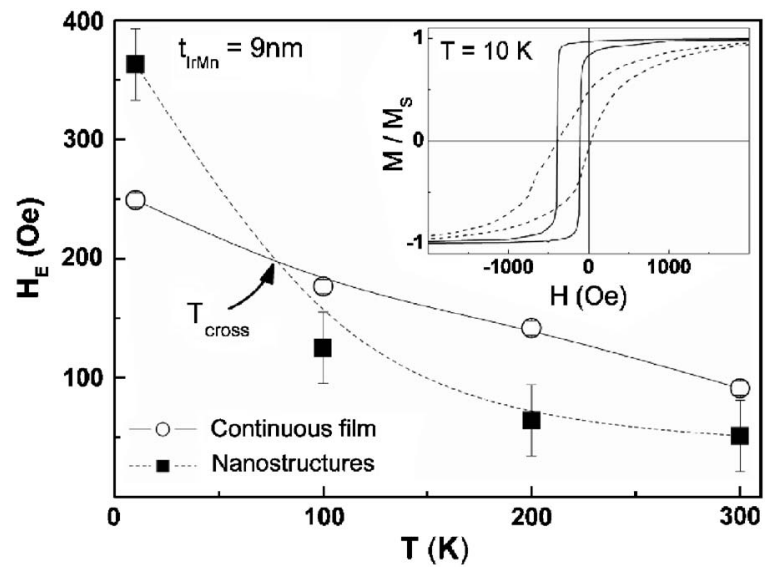

FIG. 5. Dependence of the exchange bias field $H_{E}$ on the temperature $T$ for both the continuous films (-O-) and the nanostructures (-口-), with compositions $\mathrm{Ta}(5 \mathrm{~nm}) / \mathrm{Py}(12 \mathrm{~nm}) / \operatorname{IrMn}(9 \mathrm{~nm}) /$ $\operatorname{Pt}(2 \mathrm{~nm})$. The lines are guides to the eye. The inset shows the corresponding hysteresis loops measured at $10 \mathrm{~K}$ by SQUID along the field cooling direction, after cooling from $T=550 \mathrm{~K}$ in the presence of a $2.4 \mathrm{kOe}$ field.

Figure 5 shows the evolution of $H_{E}$ on the temperature $T$ for low temperatures ranging from 10 to $300 \mathrm{~K}$, deduced from hysteresis loops measured by SQUID (a typical one is shown in the inset for $T=10 \mathrm{~K}$ ), for both continuous film and nanostructures with composition $\mathrm{Ta}(5 \mathrm{~nm}) / \mathrm{Py}(12 \mathrm{~nm}) / \mathrm{IrMn}(9 \mathrm{~nm}) / \mathrm{Pt}(2 \mathrm{~nm})$. In contrast to longitudinal Kerr effect measurements, SQUID magnetometry measurements probe both the signal from the dots and that from the trenches, i.e., from material deposited between the dots. However, the two magnetic contributions are well enough separated, hence making it possible to roughly estimate the exchange bias from the nanostructures. The loop corresponding to the patterned array (inset of Fig. 5) shows that the ascending branches of the dots and the trenches virtually match. On the contrary, the descending branch clearly exhibits two steps. The larger step (about $3 / 4$ of the signal, in agreement with the expected signals ratio) corresponds to the signal from the trenches, and the smaller step, at higher negative fields corresponds to the signal from the dots, which is the signal of interest here. It is noteworthy that, at $10 \mathrm{~K}$, for $t_{\mathrm{IrMn}}=9 \mathrm{~nm}$, the hysteresis loop of the continuous film is shifted towards lower values than the hysteresis loop of the dots, in contrast to the behavior reported in Fig. 5, and in Fig. 4(a) for higher temperatures.

As already discussed, the decrease of $H_{E}$ with $T$ for both the continuous film and the nanostructures shown in Fig. 5 can be ascribed to thermally induced weakening of AFM spins pinning strength when $T$ increases, effect which is still visible at lower sweep field rate with comparison to the sweep field rate used for MOKE measurements. The fact that $H_{E}$ decreases faster in the nanostructures confirms that nanostructured AFM layers are more subject to thermal activation effects than continuous films. This is in agreement with some results reported in the literature on low-temperature measurements of FM-AFM exchange biased nanostructures, which also show that $H_{E}$ decreases faster as $T$ is increased for confined AFM layers than for continuous films. ${ }^{11}$ It is remark-

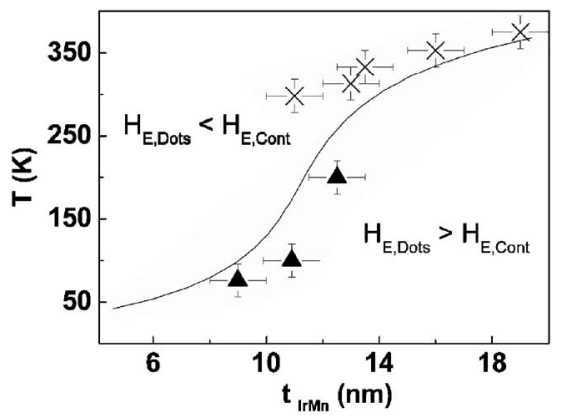

FIG. 6. Phase diagram showing the temperature $T$ and IrMn thickness $t_{\mathrm{IrMn}}$ conditions for which the exchange bias field of sub$100 \mathrm{~nm}$ nanostructures $\left(H_{E \text {,dots }}\right)$ is either larger or smaller than that of continuous films $\left(H_{E \text {,cont }}\right)$ with the same composition, at the same temperature. Note that the $(-\mathrm{x}-)$ data is deduced from room temperature Kerr effect measurements after the cooling in negative field

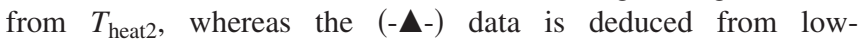
temperature SQUID measurements. The line is a guide to the eye.

able that, when thermal activation effects are minimized, the nanostructures with thin AFM layers can exhibit larger exchange bias than the continuous films, as it occurs at high temperatures for the nanostructures with thicker AFM layers (see in Fig. 4). This is in agreement with Malozemoff's static model predictions, which neglects the influence of thermal activation and predicts larger exchange bias for smaller AFM domain size. ${ }^{4}$

From the evolutions of $H_{E}$ on $T$ and $t_{\mathrm{IrMn}}$, it is possible to extract the conditions for which $H_{E}$ in the dots, $H_{E \text {,dots }}$ is larger or smaller than $H_{E}$ in the continuous films $H_{E \text {,cont }}$. This is shown in Fig. 6. The data plotted in the figure have been deduced from the evolutions of $H_{E}$ on $T_{\text {heat } 2}$ and $t_{\mathrm{IrMn}}$ (i.e., Fig. 4), from low-temperature evolutions of $H_{E}$ on $t_{\mathrm{IrMn}}$, and from low-temperature evolutions of $H_{E}$ on $T$ (Fig. 5). For instance, from Fig. 4, the value of $t_{\mathrm{IrMn}}$ at which the evolutions of $H_{E}$ on $t_{\mathrm{IrMn}}$ for the nanostructures and the continuous film cross $\left(t_{\mathrm{IrMn}, \text { cross }}\right)$ for a given $T_{\text {heat } 2}$, shows the transition from $H_{E \text {,dots }}>H_{E \text {,cont }}$ to $H_{E \text {,dots }}<H_{E \text {,cont }}$, and is then plotted in Fig. 6. Similarly, from Fig. 5, the value of $T$ at which the evolutions of $H_{E}$ on $T$ for the nanostructures and the continuous film intersect is also plotted in Fig. 6. This allows depicting a phase diagram, which summarizes the trends discussed above and can be understood with having in mind that the AFM spin structure is more prone to thermal activation for thin AFM layers and for the nanostructures. Note however that different field sweep rates were used for SQUID (about $0.03 \mathrm{Oe} \mathrm{s}^{-1}$ ) and MOKE (about $1 \mathrm{Oe} \mathrm{s}^{-1}$ ) measurements. Since thermal activation effects are more effective for the nanostructures, one would expect that $H_{E}$ decreases slightly faster with the field sweep rate for the nanostructures with respect to the continuous film, resulting in a slight overestimation of $T_{\text {cross }}$ as determined from SQUID measurements (see Fig. 5), i.e., at low field sweep rate, compared to that which would be measured using a higher rate, by MOKE.

Note that the magnetostatic energy of the FM layer in patterned EB bilayers might change the FM domain wall density during the reversal of the FM layer thus influencing $H_{C}$ and $H_{E}{ }^{19-22,40}$ The small size of the dots $(90 \mathrm{~nm})$ did not 
allow us to determine by MFM the exact reversal process of the FM. However, from the hysteresis loops, it is very likely that the reversal occurs via coherent rotation of the FM, or via the formation of high remanence states, but not via the formation of vortex or pronounced low remanence states. ${ }^{19-22}$ It has been theoretically shown that for $100 \mathrm{~nm}$ lateral dot size and $t_{\mathrm{Py}}=10 \mathrm{~nm}$, in the case of high remanence configurations, magnetostatic interactions can enhance $H_{E}$ by $10 \%$ for the nanostructures with respect to continuous films. ${ }^{40}$ It is thus not to be excluded that magnetostatic interactions contribute to the $H_{E}$ enhancement in the nanostructures. Note, however, that some AFM spins are certainly dragged during the magnetic reversal of the FM layer. Remark as well that at low temperatures (see Fig. 5), when thermal activation effects, which tend to reduce $H_{E}$ in the nanostructures are minimized, $H_{E}$ in the nanostructures is enhanced by more than $40 \%$. It is thus very likely that other effects such as, for example, AFM domain constraints previously discussed, contribute, to a large extent, to the enhancement of $H_{E}$ in the nanostructures.

It is noteworthy that our results might shed light on some of the opposite behaviors reported in the literature. For example, Ref. 8 reports an enhancement of $H_{E}$ in nanostructured $\mathrm{CoO}$ whereas Ref. 18 shows the opposite behaviour. Indeed, this might be ascribed to both the use of thicker $\mathrm{CoO}$ layer $(10 \mathrm{~nm})$ and higher temperature $(77 \mathrm{~K})$ in Ref. 8 than in Ref. 18 ( $3 \mathrm{~nm}, 5 \mathrm{~K})$. Similarly, for $\mathrm{NiO},{ }^{10-12}$ a reduction of $H_{E}$, in the nanostructures with respect to continuous films, is observed for thin AFM layers ${ }^{12}$ and an enhancement is observed for thicker AFM layers. ${ }^{10,11}$ Likewise our results on thermal activation in exchange biased nanostructures are consistent with previous works, and might thus contribute to better understand some of the reported temperature dependant evolution of $H_{E}$ in nanostructures, such as, for example, Refs. 11 and 12.

\section{CONCLUSION}

In conclusion, pronounced thermal activation effects have been observed in sub-100 nm Py/IrMn nanostructures compared with continuous films with the same composition. In addition, thinner AFM layers are found to be more prone to thermal activation. These results are ascribed to the spatial constraints imposed on the formation of AFM domains, which probably result in a loosely pinned AFM spin structure. As a result of the interplay between thermal activation effects, favoring a reduction of $H_{E}$ in the nanostructures, and the AFM domain size reduction imposed by the reduced lateral dimensions of the nanostructures, which favor the opposite behavior, the hysteresis loop shift for the nanostructures can be either larger or smaller than that of continuous films with the same composition, depending on both the AFM layer thickness and temperature conditions.

\section{ACKNOWLEDGMENTS}

This work was supported by the European Community through the NEXBIAS Grant No. HPRN-CT-2002-00296. The authors acknowledge S. Auffret and J.-F. Jacquot for their technical assistance. We are also grateful to J. Nogués for the critical reading of the manuscript and the concomitant fruitful discussions.
*Electronic mail: baltz@drfmc.ceng.cea.fr

${ }^{1}$ For recent reviews on exchange bias, see J. Nogués and I. K. Schuller, J. Magn. Magn. Mater. 192, 203 (1999); A. E. Berkowitz and K. Takano, ibid. 200, 552 (1999); R. L. Stamps, J. Phys. D 33, R247 (2000); M. Kiwi, J. Magn. Magn. Mater. 234, 584 (2001).

${ }^{2}$ D. Mauri, H. C. Siegmann, P. S. Bagus, and E. Kay, J. Appl. Phys. 62, 3047 (1987).

${ }^{3}$ A. P. Malozemoff, Phys. Rev. B 35, R3679 (1987); 37, 7673 (1988).

${ }^{4}$ A. Scholl, F. Nolting, J. W. Seo, H. Ohldag, J. Stöhr, S. Raoux, J.-P. Locquet, and J. Fompeyrine, Appl. Phys. Lett. 85, 4085 (2004).

${ }^{5}$ H. Ohldag, A. Scholl, F. Nolting, E. Arenholz, S. Maat, A. T. Young, M. Carey, and J. Stöhr, Phys. Rev. Lett. 91, 017203 (2003).

${ }^{6}$ B. Dieny, V. S. Speriosu, S. S. P. Parkin, B. A. Gurney, D. R. Wilhoit, and D. Mauri, Phys. Rev. B 43, 1297 (1991).

${ }^{7}$ S. Tehrani, J. M. Slaughter, M. Deherrera, B. N. Engel, N. D. Rizzo, J. Slater, M. Durlam, R. W. Dave, J. Janesky, B. Butcher, K. Smith, and G. Grynkewich, Proc. IEEE 91, 703 (2003).

${ }^{8}$ T. Kimura, G. Mozumi, F. Wakaya, and K. Gamo, Jpn. J. Appl. Phys., Part 1 40, 2241 (2001).

${ }^{9}$ K. Liu, S. M. Baker, M. Tuominen, T. P. Russell, and I. K.
Schuller, Phys. Rev. B 63, 060403(R) (2001).

${ }^{10}$ J. C. Wu, H. W. Huang, C. H. Lai, and T. H. Wu, J. Appl. Phys. 87, 4948 (2000).

${ }^{11}$ A. Nemoto, Y. Otani, S. G. Kim, K. Fukamichi, O. Kitakami, and Y. Shimada, Appl. Phys. Lett. 74, 4026 (1999).

${ }^{12}$ M. Fraune, U. Rüdiger, G. Güntherodt, S. Cardoso, and P. Freitas, Appl. Phys. Lett. 77, 3815 (2000).

${ }^{13}$ J. Sort, H. Glaczynska, U. Ebels, B. Dieny, M. Giersig, and J. Rybczynski, J. Appl. Phys. 95, 7516 (2004).

${ }^{14}$ V. Baltz, J. Sort, B. Rodmacq, B. Dieny, and S. Landis, Appl. Phys. Lett. 84, 4923 (2004).

${ }^{15}$ J. Yu, A. D. Kent, and S. S. P. Parkin, J. Appl. Phys. 87, 5049 (2000).

${ }^{16}$ Y. Shen, Y. Wu, H. Xie, K. Li, J. Qiu, and Z. Guo, J. Appl. Phys. 91, 8001 (2002).

${ }^{17}$ Z. B. Guo, K. B. Li, G. C. Han, Z. Y. Liu, P. Luo, and Y. H. Wu, J. Magn. Magn. Mater. 251, 323 (2002).

${ }^{18}$ E. Girgis, R. D. Portugal, H. Loosvelt, M. J. Van Bael, I. Gordon, M. Malfait, K. Temst, C. Van Haesendonck, L. H. A. Leunissen, and R. Jonckheere, Phys. Rev. Lett. 91, 187202 (2003).

${ }^{19}$ J. Eisenmenger, Z.-P. Li, W. A. A. Macedo, and I. K. Schuller, Phys. Rev. Lett. 94, 057203 (2005).

${ }^{20}$ Z.-P. Li, O. Petracic, J. Eisenmenger, and I. K. Schuller, Appl. Phys. Lett. 86, 072501 (2005). 
${ }^{21}$ J. Sort, A. Hoffmann, S. H. Chung, K. Buchanan, V. Novosad, M. Grimsditch, M. D. Baró, B. Dieny, and J. Nogués (unpublished).

${ }^{22}$ A. Hoffmann, M. Grimsditch, J. E. Pearson, J. Nogués, W. A. A. Macedo, and I. K. Schuller, Phys. Rev. B 67, 220406(R) (2003).

${ }^{23}$ V. Baltz, J. Sort, S. Landis, B. Rodmacq, and B. Dieny, Phys. Rev. Lett. 94, 117201 (2005).

${ }^{24}$ T. Eimüller, T. Kato, T. Mitzuno, S. Tsunashima, C. Quitmann, T. Ramsvik, S. Iwata, and G. Schütz, Appl. Phys. Lett. 85, 2310 (2004).

${ }^{25}$ T. Ambrose and C. L. Chien, J. Appl. Phys. 83, 6822 (1998).

${ }^{26}$ W. Pan, N.-Y. Jih, C.-C. Kuo, and M.-T. Lin, Appl. Phys. Lett. 95, 7297 (2004).

${ }^{27}$ J. P. Nozières, S. Jaren, Y. B. Zhang, A. Zeltser, K. Pentek, and V. S. Speriosu, J. Appl. Phys. 87, 3920 (2000).

${ }^{28}$ T. Hughes, K. O'Grady, H. Laidler, and R. W. Chantrell, J. Magn. Magn. Mater. 235, 329 (2001).

${ }^{29}$ A. Hoffmann, Phys. Rev. Lett. 93, 097203 (2004).

${ }^{30}$ S. Soeya, S. Nakamura, T. Imagawa, and S. Narishige, J. Appl. Phys. 77, 5838 (1995); S. Soeya, T. Himagawa, K. Mitsuoka, and S. Narishige, ibid. 76, 5356 (1994).

${ }^{31}$ M. Ali, C. H. Marrows, M. Al-Jawad, B. J. Hickey, A. Misra, U. Nowak, and K. D. Usabel, Phys. Rev. B 68, 214420 (2003).

${ }^{32}$ E. Fulcomer and S. H. Charap, J. Appl. Phys. 43, 4184 (1972); 43, 4190 (1972).

${ }^{33}$ H. Xi, J. Magn. Magn. Mater. 288, 66 (2005).

${ }^{34}$ M. D. Stiles and R. D. McMichael, Phys. Rev. B 60, 12950 (1999).

${ }^{35}$ R. L. Stamps and L. Wee, IEEE Trans. Magn. 36, 3170 (2000).

${ }^{36}$ S. Landis, B. Rodmacq, and B. Dieny, Phys. Rev. B 62, 12271 (2000).

${ }^{37}$ J. I. Martin, J. Nogués, K. Liu, J. L. Vicent, and I. K. Schuller, J. Magn. Magn. Mater. 256, 449 (2003).

${ }^{38}$ R. Skomski, J. Phys.: Condens. Matter 15, R841 (2003).

${ }^{39}$ C. Leighton, M. R. Fitzsimmons, A. Hoffmann, J. Dura, C. F. Majkrzak, M. S. Lund, and I. K. Schuller, Phys. Rev. B 65, 064403 (2002).

${ }^{40}$ S. Zhang and Z. Li, Phys. Rev. B 65, 054406 (2001). 